\title{
Can tuberculosis patients in resource-constrained settings afford chest radiography?
}

\author{
To the Editor:
}

Even when tuberculosis (TB) care is free, impoverished patients and their households continue to incur unmanageable costs due to seeking and staying in care for the full duration of anti-TB treatment [1]. By aggravating household vulnerability, these costs can prevent or delay diagnosis, treatment and successful outcome, leading to increased TB transmission, morbidity and mortality [2-4]. The new World Health Organization (WHO)'s End TB Strategy places greater emphasis on ensuring universal free access to care, and it includes a target of elimination of associated catastrophic costs for TB patients and their households by 2020 [5].

Data from prevalence surveys has led to a renewed interest in chest radiography as a triage test and a tool for active case finding $[6,7]$. Today chest radiography is employed in many settings both for screening and as part of the diagnostic algorithm and follow-up. However, if chest radiography is not provided free of charge to patients, there is a risk that costs incurred by patients during their path to a TB diagnosis may be aggravated.

We sought to provide a snapshot of current accessibility of chest radiography to TB patients and patients accessing care with symptoms suggestive of TB (presumptive TB cases [8]), both geographically and financially. We consulted experts from 44 low- and middle-income countries (of these, 12 are from the 30 countries deemed to have a high TB burden and 10 are from the 30 countries deemed to have a high burden of multidrug resistant (MDR)-TB (figure 1)), using an online survey with open and multiple-choice questions. The questionnaire was sent via email to staff working in national TB programmes (NTPs) or consultants and researchers working closely with NTPs. We received a response from 27 (61.4\%) of them.

The survey asked about the availability and cost of chest radiography in public health facilities, role of chest radiography in the country's diagnostic algorithm, provision of health insurance or other forms of social protection to TB patients and presumptive TB cases (table 1).

In most countries (19 out of 27), chest radiography is part of the routine diagnostic algorithm and follow-up. In over half of the countries (15 (55\%) out of 27$)$ patients have to pay for a chest radiography examination in the public health service. The cost for a chest radiograph varies between USD 1.5 and USD 42 (median USD 7.8). The highest costs were reported from African countries (figure 1). Costs were reported to vary even within countries: prices ranged between USD 8.4 and USD 42 in Senegal, and between USD 5 and USD 20 in Zimbabwe, dependent on the facility and the region. Some patients were exempt from payment: MDR-TB patients (Bolivia, Burkina Faso and Senegal), patients co-infected with HIV (Ghana and Burkina Faso), children aged $<5$ years (Bolivia, Burkina Faso and Vietnam), patients categorised as very poor by their kebele ("neighbourhood") leaders (Ethiopia) and patients under follow-up (Kyrgyz Republic). In contrast, sputum smear microscopy is provided free of charge in all countries. In countries where chest radiographs are also employed for monitoring progress during treatment, this exposes patients to even higher costs, as they must pay for diagnostic chest radiography and also follow-up chest radiography examinations.

TB diagnostics and treatment is free of charge with the exception of chest radiographs in most low-income countries without a national health insurance scheme (such as Zimbabwe). The same holds true for middle-income countries (Vietnam, Dominican Republic, Indonesia and Peru). However, those countries often have health insurance schemes covering the costs of chest radiographs. Health insurance schemes

@ERSpublications

In many low- and middle-income countries chest radiographs are not free of charge for TB patients http://ow.ly/SA7l305Yzk2

Cite this article as: Pedrazzoli D, Lalli M, Boccia D, et al. Can tuberculosis patients in resource-constrained settings afford chest radiography? Eur Respir J 2017; 49: 1601877 [https://doi.org/ $10.1183 / 13993003.01877-2016]$. 


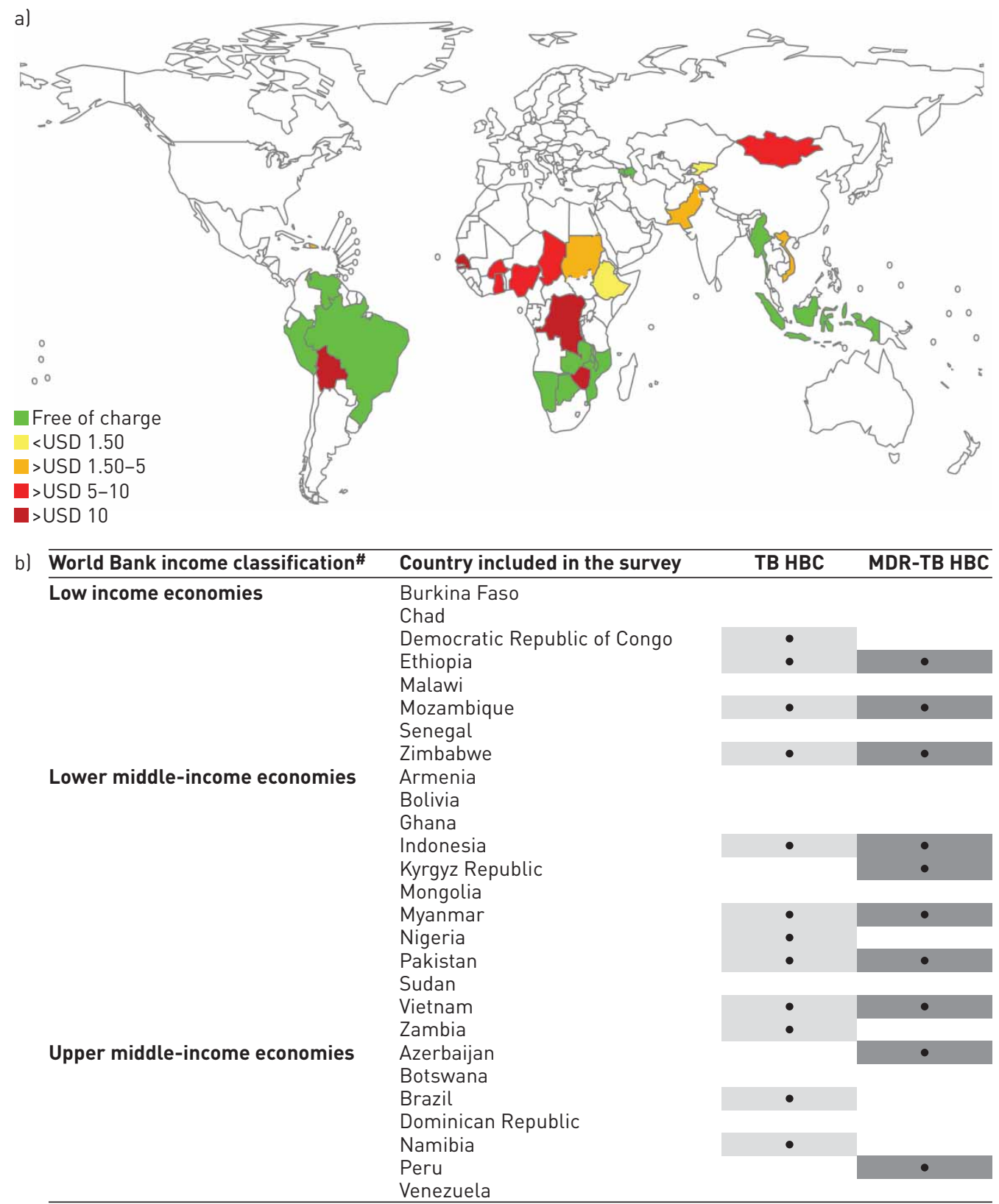

FIGURE 1 Costs of tuberculosis (TB). a) Cost of chest radiography; b) inclusion by the World Health Organization on list of high-burden countries (HBC) for TB and multidrug-resistant (MDR)-TB for the period 2016-2020 [9]. \#: World Bank country classification by income: low-income economies are defined as those with a gross national income (GNI) per capita, calculated using the World Bank Atlas method, of SUSD 1025 in 2015; lower middle-income economies are those with a GNI per capita of USD 1026-4035; and upper middle-income economies are those with a GNI per capita of USD 4036-12475.

based on a contributory model (such as in Ghana) are only accessible to individuals employed in the formal sector. Hence the poorest, who are at greatest risk of TB, are left out of the health insurance scheme and are unable to access chest radiography free of charge.

Our analysis has limitations. It is likely that we underestimated the costs of chest radiography for patients as we only considered direct medical costs, but hidden direct "out of pocket" costs such as transport costs (especially when chest radiography facilities are not on site) and food, as well as indirect costs due to loss of productivity, tend to account for a sizable proportion of expenditure on seeking and receiving care [1]. Patient costs surveys that are currently under way will provide useful insights and more comprehensive estimates.

We focused on the public sector only. The private sector often represents the first point of care for most TB patients in many Asian countries [8]. Data reported from Pakistan show that chest radiography costs 
TABLE 1 Main topics and domains covered by the survey questionnaire

Where can patients get a chest radiography, e.g. public hospital, private provider? Is chest radiography free in the public service?

If chest radiography is not provided free of charge, how much does it cost?

Are there certain circumstances when chest radiography is provided free of charge, e.g. for children?

Is chest radiography part of the diagnostic algorithm in your country?

Is smear microscopy provided free of charge in the public service in your country?

Are TB patients in your country covered by a national health insurance scheme or other forms of social protection le.g. cash transfer to cover the cost of TB diagnosis and treatment)?

If so, does the national health insurance cover the cost of chest radiography for TB patients?
Coverage and access to chest radiography

Access to and cost of chest radiography

Cost of chest radiography

Cost of chest radiography

Role of chest radiography in diagnostic algorithm

Cost of diagnosis by smear microscopy

Availability of social protection/health insurance in the country

Coverage of TB patients by health insurance

TB: tuberculosis.

in the private sector can be up to four times higher than in public health facilities (USD 2.5-8.0 versus USD 1.5-2.0).

Our survey is not representative of the global level, nor was it intended to be. However, we covered 12 out of the 30 high TB burden and 10 out of the 30 high MDR-TB burden countries. Furthermore, we included countries from the three most affected continents (figure 1).

As prevalence surveys have shown, expanding the use of chest radiography has a great potential as a screening/triage tool and can contribute to achieving the ambitious targets set in the End TB Strategy [6]. However, the TB community needs to be aware that widespread use of chest radiography might potentially aggravate costs for patients and presumptive TB cases. Chest radiography, similar to sputum smear microscopy and Xpert MTB/RIF (Cepheid, Sunnyvale, CA, USA), should be easily accessible and free of charge if employed for TB diagnosis and follow-up. A recently published WHO policy document on chest radiography aptly states that chest radiographs should be free of charge and/or fully reimbursed by health insurance [10]. Additionally, practical approaches to mitigate patient costs should be provided to NTPs. Harmonisation within and across countries and donors will be necessary. Another potential avenue to reducing the costs for patients is the extension of social protection interventions (such as cash transfers and health insurance) to cover all costs associated with TB diagnosis and treatment. This will require strong political commitment and dedicated resources, and it will be difficult to implement in the near future.

In conclusion, the direct costs of chest radiography for patients are high. In many of the countries participating in this survey a large proportion of their population lives on $<$ USD 1.9 per day [11]. Efforts are made to reduce financial barriers for patients by providing smear microscopy, Xpert MTB/RIF and treatment free of charge. A similar approach is necessary if chest radiographs become part of the diagnostic algorithms.

Debora Pedrazzoli $^{1,2}$, Marek Lalli ${ }^{1,2}$, Delia Boccia ${ }^{2}$, Rein Houben ${ }^{1,2}$ and Katharina Kranzer ${ }^{2,3}$

${ }^{1}$ TB Modelling Group, TB Centre and CMMID, London School of Hygiene and Tropical Medicine, London, UK. ${ }^{2}$ Department of Infectious Disease Epidemiology, London School of Hygiene and Tropical Medicine, London, UK. ${ }^{3}$ National and Supranational Reference Laboratory, Research Centre Borstel, Borstel, Germany.

Correspondence: Debora Pedrazzoli, Faculty of Epidemiology and Population Health, London School of Hygiene and Tropical Medicine, Keppel Street, London, WC1E 7HT, UK. E-mail: debora.pedrazzoli@lshtm.ac.uk

Received: Oct 062016 | Accepted after revision: Oct 252016

Conflict of interest: None declared.

Acknowledgements: We would like to thank all the country experts that contributed information to this study. In addition, we would like to thank Andrew Codlin (Stop TB Partnership, Geneva, Switzerland) and Andrew Siroka (WHO/Global TB Programme, Geneva, Switzerland) for their help with providing contacts in countries.

\section{References}

1 Tanimura T, Jaramillo E, Weil D, et al. Financial burden for tuberculosis patients in low-and middle-income countries: a systematic review. Eur Respir J 2014; 43: 1763-1775.

2 Laurence Y, Griffiths U, Vassall A. Costs to health services and the patient of treating tuberculosis: a systematic literature review. Pharmacoeconomics 2015; 33: 939-955. 
3 Ukwaja KN, Alobu I, Lgwenyi C, et al. The high cost of free tuberculosis services: patient and household costs associated with tuberculosis care in Ebonyi State, Nigeria. PloS One 2013; 8: e73134.

4 Wingfield T, Boccia D, Tovar M, et al. Defining catastrophic costs and comparing their importance for adverse tuberculosis outcome with multi-drug resistance: a prospective cohort study, Peru. PLoS Med 2014; 11: e1001675.

World Health Organization. The End TB Strategy. Geneva, World Health Organization, 2015.

6 World Health Organization. Chest Radiography for Tuberculosis Detection. www.who.int/tb/publications/ Radiography_TB_factsheet.pdf?ua=1 Date last accessed: June 4, 2016. Date last updated: 2016.

7 World Health Organization. Tuberculosis Prevalence Surveys: a Handbook. Geneva, World Health Organization, 2011.

8 Hazarika I. Role of private sector in providing tuberculosis care: evidence from a population-based survey in India. J Glob Infect Dis 2011; 3: 19-24.

9 World Health Organization. Use of High Burden Country Lists for TB by WHO in the Post-2015 Era. Geneva, World Health Organization, 2015.

10 World Health Organization. Chest Radiography in Tuberculosis Detection - Summary of WHO Recommendations and Guidance on Programmatic Approaches. Geneva, World Health Organization, 2016.

11 World Bank. Poverty \& Equity Data. Date last accessed: September 1, 2016. Date last updated: 2016.

Copyright @eERS 2017 